De Tampere à Séville : bilan de la sécurité européenne $(1 / 2)$

\title{
Conclusions de la présidence du conseil européen de Séville 21 et 22 juin 2002 (extraits)
}

\section{Union européenne}

\section{(2) OpenEdition \\ Journals}

Édition électronique

URL : http://journals.openedition.org/conflits/791

DOI : $10.4000 /$ conflits. 791

ISSN : $1777-5345$

Éditeur :

CCLS - Centre d'études sur les conflits lilberté et sécurité, L'Harmattan

Édition imprimée

Date de publication : 1 mars 2002

ISBN : 2-7475-3029-9

ISSN : 1157-996X

\section{Référence électronique}

Union européenne, «Conclusions de la présidence du conseil européen de Séville 21 et 22 juin 2002 (extraits) », Cultures \& Conflits [En ligne], 45 | printemps 2002, mis en ligne le 22 mars 2006, consulté le 30 mars 2021. URL : http://journals.openedition.org/conflits/791 ; DOI : https://doi.org/10.4000/ conflits. 791

Ce document a été généré automatiquement le 30 mars 2021.

Creative Commons License 


\title{
Conclusions de la présidence du conseil européen de Séville 21 et 22 juin 2002 (extraits)
}

\author{
Union européenne
}

D/02/13 Bruxelles, le 22 juin 2002

2 Le Conseil européen s'est réuni à Séville les 21 et 22 juin 2002. La réunion a été précédée par un exposé du président du Parlement européen, M. Pat Cox, suivi d'un échange de vues sur les principaux sujets à l'ordre du jour. (...)

4 Le Conseil européen a approuvé le rapport de la présidence concernant la Politique de sécurité et de défense.

5 Le Conseil européen, déterminé à renforcer le rôle de l'Union européenne dans la lutte contre le terrorisme et reconnaissant l'importance de la contribution de la PESC, y inclus la PESD, à cette fin, a adopté une Déclaration (voir Annexe V) destinée à prendre plus en considération les capacités requises pour combattre le terrorisme.

6 À la suite de la déclaration d'opérationnalité de la PESD adoptée à Laeken, des progrès substantiels ont été faits concernant le développement des capacités civiles et militaires, la mise en œuvre du plan d'action destiné à combler les lacunes existantes, et les perspectives de la coopération en matière d'armement. Le Conseil européen demande aux ministres de la défense, au Conseil " Affaires générales ", de continuer à orienter le développement de ces travaux sur les capacités.

7 L'Union européenne a confirmé qu'elle était en mesure de prendre en charge des opérations de gestion de crise, en décidant notamment de conduire la mission de police en Bosnie Herzégovine (EUPM), qui assurera le suivi, à partir du 1 janvier 2003, de l'actuelle opération de l'ONU.

8 Le Conseil européen a exprimé la volonté de l'Union européenne d'assurer la relève de l'OTAN dans l'ancienne République yougoslave de Macédoine. Il a chargé le Secrétaire général/Haut représentant et les instances compétentes de l'Union européenne de 
prendre les contacts nécessaires avec les autorités de l'ancienne République yougoslave de Macédoine et les responsables de l'OTAN, et de poursuivre et intensifier les mesures de planification entreprises, de manière à être en mesure d'assurer la relève de l'opération de l'OTAN à l'issue du mandat actuel de cette dernière, étant entendu que les arrangements permanents entre l'Union européenne et l'OTAN (Berlin+) seraient alors en place.

9 Se félicitant des progrès accomplis jusqu'à présent par la Présidence espagnole concernant la mise en œuvre des dispositions du traité de Nice relatives à la participation des alliés européens non membres de l'Union européenne, le Conseil européen charge la prochaine présidence, ainsi que le Secrétaire général/Haut Représentant, de poursuivre ces travaux.

10 Dans le domaine civil, le travail s'est poursuivi dans les quatre secteurs prioritaires (police, état de droit, administration civile et protection civile) tant sur les aspects qualitatifs que quantitatifs des capacités. Les structures et procédures de décision de la PESD ont été testées avec succès lors du premier exercice de gestion de crise conduit par l'Union.

11 Un rapport sur l'ensemble de ces sujets sera présenté au Conseil européen de Copenhague. (...)

Le Conseil européen est déterminé à accélérer la mise en œuvre, sous tous ses aspects, du programme adopté à Tampere en vue de la création d'un espace de liberté, de sécurité et de justice dans l'Union européenne. Dans ce contexte, le Conseil européen rappelle la nécessité de développer une politique commune de l'Union européenne sur les questions, distinctes mais étroitement liées de l'asile et de l'immigration.

Il est capital pour l'Union européenne et ses Etats membres que les flux migratoires soient gérés dans le respect du droit, en coopération avec les pays d'origine et de transit de ces flux. Le Conseil européen accueille donc avec satisfaction les résultats obtenus au cours du semestre écoulé, notamment le Plan global de lutte contre l'immigration clandestine et le Plan pour la gestion des frontières extérieures et la directive établissant les normes minimales pour l'accueil des demandeurs d'asile dans les Etats membres, et invite les prochaines présidences à continuer de donner aux questions de migration une place privilégiée dans les calendriers des travaux.

15 Les mesures prises à court et moyen terme pour la gestion conjointe des flux migratoires doivent respecter un juste équilibre entre, d'une part, une politique d'intégration des immigrants légalement établis et une politique d'asile qui respecte les conventions internationales, principalement la Convention de Genève de 1951, et, d'autre part, une lutte résolue contre l'immigration clandestine et la traite des êtres humains.

L'action de l'Union dans ce domaine doit se fonder sur les principes suivants :

l'aspiration légitime à une vie meilleure doit être conciliable avec la capacité d'accueil de l'Union et de ses Etats membres et l'immigration doit être canalisée dans les voies légales prévues à cet effet; l'intégration des immigrants en situation régulière dans l'Union entraîne à la fois des droits et des obligations au regard des droits fondamentaux reconnus dans l'Union; dans ce contexte, la lutte contre le racisme et la xénophobie revêt une importance essentielle ; 

contribuer, entre autres, à une meilleure maîtrise des flux migratoires. Il demande de mettre en place sans délai, dans le cadre du Conseil, l'instance commune de praticiens des frontières extérieures, composée des chefs des services de contrôle aux frontières des Etats membres et chargée de cordonner les mesures figurant dans le plan. Il 
demande par ailleurs au Conseil, à la Commission et aux Etats membres, chacun dans le cadre de ses compétences, de mettre en œuvre les mesures suivantes : effort accru de la part de l'Union européenne et une approche ciblée du phénomène, en utilisant tous les instruments appropriés dans le cadre des relations extérieures de l'Union européenne. À cet effet, et en accord avec les conclusions du Conseil de Tampere, une approche intégrée, globale et équilibrée visant à s'attaquer aux causes profondes de l'immigration illégale doit rester l'objectif constant de l'Union européenne à long terme. Dans cette optique, le Conseil européen rappelle que l'intensification de la coopération économique, le développement des échanges commerciaux, l'aide au développement, ainsi que la prévention des conflits, sont autant de moyens de favoriser la prospérité économique des pays concernés et, de ce fait, de réduire les causes qui sont à l'origine des mouvement migratoires. Le Conseil européen demande instamment que, dans tout futur accord de coopération, accord d'association ou accord équivalent que l'Union européenne ou la Communauté européenne conclura avec quelque pays que ce soit, soit insérée une clause sur la gestion conjointe des flux migratoires ainsi que sur la réadmission obligatoire en cas d'immigration illégale. et de transit en matière de gestion conjointe et de contrôle des frontières ainsi qu'en matière de réadmission. Cette réadmission par les pays tiers inclura celle de leurs propres ressortissants présents illégalement dans un Etat membre, ainsi que, dans les mêmes conditions, la réadmission des ressortissants de pays tiers dont le transit par le pays en question peut être établi. La coopération vise à obtenir des résultats à court et moyen terme. L'Union est disposée à fournir l'aide technique et financière nécessaire pour ce faire, auquel cas la Communauté européenne devra se voir accorder les ressources appropriées, dans le cadre des perspectives financières.

Le Conseil européen estime qu'il est nécessaire de procéder à une évaluation systématique des relations avec les pays tiers qui ne coopèrent pas dans la lutte contre l'immigration illégale. Cette évaluation sera prise en compte dans les relations entre l'Union européenne et ses Etats membres et les pays concernés, dans tous les domaines pertinents. Une coopération insuffisante de la part d'un pays pourrait rendre plus difficile l'approfondissement des relations entre le pays en question et l'Union. 
41 Le Conseil, après que les mécanismes communautaires existants auront été épuisés sans résultat, pourra constater, à l'unanimité, un manque de coopération non justifié de la part d'un pays tiers dans la gestion commune des flux migratoires. Dans ce cas le Conseil pourra adopter, conformément aux règles des traités, des mesures ou des positions dans le cadre de la Politique étrangère et de sécurité commune et des autres politiques de l'Union européenne, tout en respectant les engagements contractés par l'Union et sans mettre en cause les objectifs de la coopération au développement.

Accélération des travaux législatifs en cours sur la définition d'une politique commune en matière d'asile et d'immigration

Parallèlement à la coopération renforcée pour lutter contre l'immigration clandestine, il convient de faire avancer l'examen des propositions en cours. Le Conseil européen demande instamment au Conseil d'approuver : avant décembre 2002, le règlement Dublin II ; avant juin 2003, les normes relatives aux conditions requises pour bénéficier du statut de réfugié et au contenu de ce statut; les dispositions sur le regroupement familial et le statut des résidents permanents de longue durée ; avant la fin de 2003, les normes communes en matière de procédures d'asile.

La Commission présentera un rapport au Conseil fin octobre sur l'efficacité des ressources financières disponibles au niveau communautaire en matière de rapatriement des immigrants et des demandeurs d'asile déboutés, de gestion des frontières extérieures et de projets d'asile et migration dans les pays tiers.

Le Conseil européen demande au Conseil de lui présenter, en collaboration avec la Commission, pour sa réunion en juin 2003, un rapport sur la mise en œuvre pratique des orientations figurant dans le présent chapitre. (...)

\section{PROJET DE DÉCLARATION DU CONSEIL EUROPÉEN SUR LA CONTRIBUTION DE LA PESC,} Y COMPRIS LA PESD, À LA LUTTE CONTRE LE TERRORISME

51 1. Le Conseil européen réaffirme que le terrorisme pose un véritable défi à l'Europe et au monde et constitue une menace pour notre sécurité et notre stabilité. C'est pour cette raison que le Conseil européen a décidé, lors de sa réunion extraordinaire du 21 septembre 2001, d'intensifier son engagement contre le terrorisme à travers une approche coordonnée et interdisciplinaire incorporant toutes les politiques de l'Union, notamment en développant la politique étrangère et de sécurité commune (PESC) et en rendant la politique européenne de sécurité et de défense (PESD) opérationnelle.

2. Le Conseil européen a pris acte des résultats importants obtenus dans la mise en œuvre du plan d'action contre le terrorisme et réaffirme que la lutte contre le terrorisme restera un objectif prioritaire de l'Union européenne et un des principaux volets de sa politique extérieure. La solidarité et la coopération internationale sont des instruments essentiels pour lutter contre ce fléau. L'Union continuera à coordonner son action aussi étroitement que possible avec les Etats-Unis et d'autres partenaires. L'Union s'efforcera de contribuer davantage encore aux efforts internationaux, tant sur le plan interne que dans ses relations avec les pays tiers et les organisations internationales telles que les Nations Unies, l'OTAN et l'OSCE.

53 3. La politique étrangère et de sécurité commune, y compris la politique européenne de sécurité et de défense, peut jouer un rôle important pour contrer cette menace pour 
notre sécurité et promouvoir la paix et la stabilité. Les Etats membres travaillent actuellement en coopération plus étroite face à la situation internationale créée par les attentats terroristes du 11 septembre.

4. Le Conseil européen se félicite des progrès accomplis depuis le 11 septembre pour ce qui est d'intégrer la lutte contre le terrorisme dans tous les aspects de la politique extérieure de l'Union. La lutte contre le terrorisme nécessite une approche globale, l'objectif étant de renforcer la coalition internationale et de prévenir ou de contenir les conflits régionaux. L'Union : renforce ses instruments pour la prévention à long terme des conflits, met l'accent, dans son dialogue politique avec les pays tiers, sur la lutte contre le terrorisme, ainsi que sur la non-prolifération et la maîtrise des armements, fournit une assistance à des pays tiers afin qu'ils soient mieux à même de réagir efficacement à la menace internationale du terrorisme, fait figurer des clauses antiterrorisme dans les accords qu'elle conclut avec les pays tiers, réévalue ses relations avec les pays tiers en fonction de leur attitude envers le terrorisme et agit en conséquence, met en œuvre des mesures spécifiques dans la lutte contre le terrorisme, conformément à la déclaration 1373 du Conseil de sécurité des Nations Unies, qui a défini une série de mesures et de stratégies de tous ordres pour lutter contre le terrorisme, y compris des mesures financières.

55 5. Le Conseil européen se félicite également des progrès réalisés dans la mise en œuvre de la PESD, à la suite de la déclaration d'opérationnalité de la politique européenne de sécurité et de défense. Ces progrès ont permis à l'Union de prendre sa première décision visant à mettre sur pied une opération de gestion de crise, la mission de police de l'Union européenne en Bosnie-Herzégovine (MPUE), qui témoigne de la volonté de l'Union européenne de stabiliser les régions sortant d'un conflit et d'apporter sa contribution à l'instauration de l'Etat de droit. En favorisant la stabilité, et notamment en renforçant les capacités locales de maintien de l'ordre, ainsi que les normes et les règles en la matière, l'Union européenne contribue à priver les organisations terroristes de la possibilité de s'enraciner. Comme indiqué lors du Conseil européen de Laeken, grâce aux capacités militaires et civiles mises en place par l'Union européenne pour la gestion des crises, la PESC deviendra plus forte et contribuera plus efficacement à la lutte contre le terrorisme dans l'intérêt des populations concernées.

6. La PESD se renforcera au fur et à mesure que les Etats membres développeront leurs capacités militaires et civiles de gestion des crises. Dans cette perspective, le Conseil européen souligne à nouveau l'importance qu'il attache à ce que les cibles de l'objectif global soient atteintes dans les délais. Dans ce contexte, le développement de la PESD doit tenir davantage compte des capacités qui pourraient être nécessaires, conformément aux missions de Petersberg et aux dispositions du traité, pour lutter contre le terrorisme.

57 7. En matière de lutte contre le terrorisme, y compris dans le domaine de la PESC et de la PESD, l'Union européenne devrait par priorité : consacrer davantage d'efforts à la prévention des conflits, approfondir le dialogue politique avec les pays tiers pour faire progresser la lutte contre le terrorisme, notamment en promouvant les droits de l'homme et la démocratie, ainsi que la non-prolifération et la maîtrise des armements, et apporter à ces pays l'aide internationale requise, renforcer les mécanismes d'échange de renseignements et recourir davantage à l'évaluation des situations et aux rapports d'alerte rapide, en se fondant sur un maximum de sources différentes, se doter d'une évaluation commune de la menace terroriste qui pèse sur les Etats 
membres ou les forces déployées en dehors de l'Union, dans le cadre de la PESD, pour des opérations de gestion de crises, y compris de la menace d'une utilisation à des fins terroristes d'armes de destruction massive, déterminer les capacités militaires nécessaires pour protéger contre des attentats les forces déployées dans le cadre d'opérations de gestion de crises menées par l'Union européenne, étudier plus avant comment des capacités militaires ou civiles pourraient être utilisées pour contribuer à protéger les populations civiles contre les effets d'attentats.

8. Le Conseil européen demande à la présidence et au Secrétaire général/Haut représentant, ainsi que, s'il y a lieu, à la Commission, d'accentuer leurs efforts dans ces domaines prioritaires en encourageant la coordination au sein des instances du Conseil et avec les organisations internationales concernées, notamment les Nations Unies et l'OTAN, afin de rendre plus efficace la contribution de la PESC, y compris celle de la PESD, dans la lutte contre le terrorisme, et de faire rapport sur ces questions au Conseil «Affaires générales ». (...)

\section{ANNEXE VIII}

\section{LISTE DES RAPPORTS DE RÉFÉRENCE/RAPPORTS DESTINÉS AU CONSEIL EUROPÉEN (...)}

Communication de la Commission au Conseil et au Parlement européen intitulée «Vers une gestion intégrée des frontières extérieures des Etats membres de l'Union européenne » [doc. 9139/02] (..)

Rapport de la présidence sur les progrès réalisés dans la lutte contre l'immigration clandestine [doc. 10009/02]

Note de la présidence sur la coopération avec les pays tiers d'origine et de transit en vue de lutter conjointement contre l'immigration clandestine [doc. 9917/3/02 REV 3]

Conclusions du Conseil JAI sur les mesures à appliquer pour prévenir et combattre l'immigration illégale ainsi que le trafic de migrants et la traite des êtres humains par voie maritime [doc. 10017/02]

Plan pour la gestion des frontières extérieures [doc. 10019/02] (...)

\section{INDEX}

Mots-clés : construction européenne, textes et documents officiels 